\title{
Toward full waveform inversion of controlled-source electromagnetic exploration to realistic scale
}

\author{
Naoto IMAMURA ${ }^{1}$, Tada-nori GOTO ${ }^{1}$, Junichi TAKEKAWA ${ }^{1}$ \\ and Hitoshi MIKADA ${ }^{1}$ \\ ${ }^{1}$ Dept. of Civil and Earth Res. Eng., Kyoto University
}

\begin{abstract}
A 3D full waveform inversion method is presented using controlled-source electromagnetic (CSEM) method. Using data from a synthetic model, we demonstrate that conductive anomalies around subsurface could be estimated. We discussed the resolution of our CSEM inversion method, considering the orientation of the dipoles of transmitter and receivers. The synthetic inversion examples show that horizontal source dipole gives high resolution for horizontal position of anomaly. We also find that vertical transmitter gives high resolution for deeper position of anomaly. When the 3-components transmitter's model gradients are considered, the inversion result have high resolution for horizontal and vertical positions. These differences of the inversion results are explained considering the orientation of electric flux. From numerical results, we consider that it is efficient to employ different oriented dipoles of transmitter and receivers for accurate inversion.
\end{abstract}

\section{INTRODUCTION}

Recently, controlled source electromagnetic (CSEM) method is widely used for shallow subsurface exploration to measure resistivity structure in detail. This method is also used for surveying oil and natural gas resources in deep sea. Various kinds of inversion methods in frequency domain have been developed, although this method requires solutions for each frequency in the source spectrum. In this paper, we present the implementation of full waveform inversion algorithm to simulate multi-frequencies at a time. For full waveform inversion method, Zhdanov and Portniaguine (1997) derived basic theory of this inversion method and confirmed the effectiveness of this inversion in their numerical study.

Zhdanov (2010) applied this inversion to real field data. From previous research, observed electromagnetic fields highly depend on the orientation of transmitter.

In time-domain electromagnetic method, FDTD method is often used for forward calculation. However, many previous researches show that when FDTD method is used with low frequency transmitter, huge number of time step is needed. In this study, we solve the problem employing fictitious wave domain method $^{3)}$. In this method, electromagnetic fields are calculated in fictitious wave domain and transformed to diffusive domain employing mathematical transform. Time step in FDTD method becomes much larger than time step derived from courant condition.

Employing these forward and inversion simulations, we present the implementation of a 3D full waveform inversion algorithm for synthetic marine CSEM data. We discussed our results in terms of resolution of orientations of transmitters and receivers and found out that the combination of orientations of transmitters and receivers is a key to determine resolution of inversion results.

\section{METHOD}

\section{(1)FDTD method in Fictitious wave domain}

Three dimensional Maxwell equations are discretised using 4th-order finite-difference scheme. Because of huge numbers of time step, it is very time consuming to simulate FDTD method with low frequency transmitter. In order to calculate FDTD method with large time step such as real field data, fictitious wave domain method is employed. Frequency domain Maxwell equations are transformed to fictitious domain employing following equation,

$$
\omega^{\prime}=(i+1) \sqrt{\omega \omega_{0}}
$$

where $\omega_{0}$ is a scale parameter. Employing this equation, frequency domain Maxwell equations becomes,

$$
\begin{array}{r}
-\nabla \times \mathbf{H}^{\prime}\left(\mathbf{x}, \omega^{\prime}\right)-i \omega^{\prime} \epsilon^{\prime}(\mathbf{x}) \mathbf{E}^{\prime}\left(\mathbf{x}, \omega^{\prime}\right)=-\mathbf{J}^{\prime}\left(\mathbf{x}, \omega^{\prime}\right), \\
\nabla \times \mathbf{E}^{\prime}\left(\mathbf{x}, \omega^{\prime}\right)-i \omega^{\prime} \mu \mathbf{H}^{\prime}\left(\mathbf{x}, \omega^{\prime}\right)=-\mathbf{K}^{\prime}\left(\mathbf{x}, \omega^{\prime}\right)
\end{array}
$$

where $\epsilon^{\prime}$ satisfy $\epsilon^{\prime}(\mathbf{x})=\sigma(\mathbf{x}) / 2 \omega_{0}$. The fields in the fictitious wave domain do not represent observable fields. Propagation paths and interaction amplitudes are not altered by the transform from the fictitious wave domain to the diffusive frequency domain. Finally, fictitious wave domain equations in time domain are de- 


\begin{tabular}{|l|c|c|}
\hline & $\mathrm{dt}(\mathrm{s})$ & Calc. time (m) \\
\hline Courant cond. (with air) & $1.9 \times 10^{-7}$ & 1230000 (est) \\
\hline Courant cond. (without air) & $1.9 \times 10^{-5}$ & 12300 (est) \\
\hline Fictitious wave domain & 0.015634 & 15 \\
\hline
\end{tabular}

Fig. 1 Time step length of fictitious wave domain method and courant condition

Received Green function

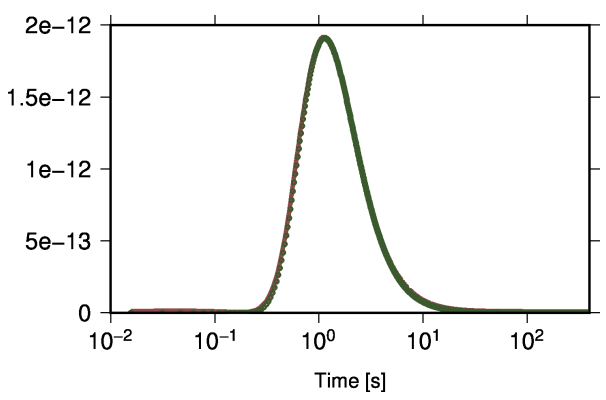

Fig. 2 Validation of forward calculation in time series.

rived as follows,

$$
\begin{aligned}
-\nabla \times \mathbf{H}^{\prime}\left(\mathbf{x}, t^{\prime}\right)+\epsilon^{\prime}(\mathbf{x}) \partial_{t^{\prime}} \mathbf{E}^{\prime}\left(\mathbf{x}, t^{\prime}\right) & =-\mathbf{J}^{\prime}\left(\mathbf{x}, t^{\prime}\right), \\
\nabla \times \mathbf{E}^{\prime}\left(\mathbf{x}, t^{\prime}\right)+\mu \partial_{t^{\prime}} \mathbf{H}^{\prime}\left(\mathbf{x}, t^{\prime}\right) & =-\mathbf{K}^{\prime}\left(\mathbf{x}, t^{\prime}\right)
\end{aligned}
$$

In this study, equation (4) and (5) are solved by FDTD method. Electromagnetic fields in diffusive domain are calculated using mathematical transform. In equation (4) and (5), velocity of electromagnetic fields are calculated from $c(\mathbf{x})=\sqrt{2 \omega_{0} / \mu \sigma(\mathbf{x})}$. Depend on the value of $\omega_{0}$, velocity of electromagnetic fields can be set optimally. In this study, we set $\omega_{0}$ as 6.28 . When $\omega_{0}$ equals to 6.28 and grid size equals to $100 \mathrm{~m}$, time step length becomes to $0.015634 \mathrm{~s}$. This value is about eight thousand times larger than a time step length based on courant condition. Detail comparison of time step length is shown in Fig.1. For FDTD schemes, the convolutional perfectly matched layer (CPML) is employed. The absorption properties of a CPML are determined by the complex stretching variable

$$
s_{i}\left(u, \omega^{\prime}\right)=\kappa_{i}(u)+\frac{\sigma_{i}(u)}{\alpha_{i}(u)+i \omega^{\prime}},(i=x, y, z)
$$

where $0<u<\delta_{\zeta}$, the function $\alpha_{\zeta}(u), \sigma_{\zeta}(u)$ and $\kappa(u)$ must be designed appropriately.

Employing this method, electromagnetic fields and green function in time domain can be calculated. Obtained green function can be validated using whole space solution of implies response of electric fields ${ }^{4)}$. Fig. 2 shows the result of validation when offset equal to $3000 \mathrm{~m}$.

\section{(2)Full waveform inversion}

Our inversion flow is shown in Fig.3. In our inversion

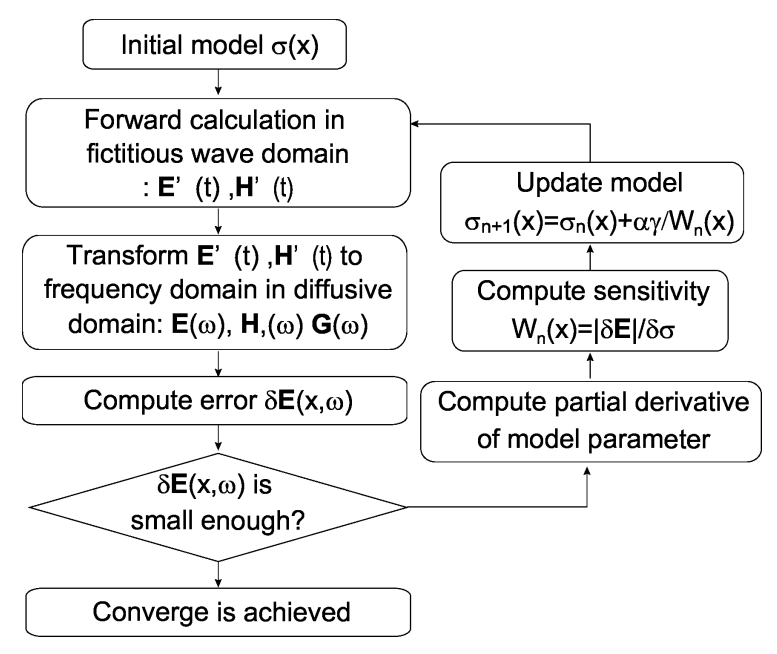

Fig. 3 Flow chart of inversion method.

method, model parameter is calculated based on,

$\sigma_{n+1}=\sigma_{n}+\alpha_{n} \mathbf{W}_{n}^{-1} \sum_{t r n} \int_{\Omega} \mathbf{E}(x, \omega) \cdot \mathbf{E}_{b}\left(x, \omega \mid \delta \mathbf{E}_{0}\right) d \omega$

where $\mathbf{E}_{b}$ and $\delta \mathbf{E}, \mathbf{W}_{n}$ are calculated from

$$
\begin{array}{r}
\mathbf{E}_{b}\left(x, \omega \mid \delta \mathbf{E}_{0}\right)=\iint_{S} \mathbf{G}^{E J}\left(x, \omega ; x_{r}\right) \cdot \delta \mathbf{E}_{0}\left(x_{r}, \omega\right) d s \\
\delta \mathbf{E}\left(x_{r}, \omega \mid x_{s}\right)=\mathbf{E}\left(x_{r}, \omega ; x_{s}\right)_{o b s}-\mathbf{E}\left(x_{r}, \omega ; x_{s}\right)_{c a l}{ }^{(8)} \\
\mathbf{W}_{n}=\sqrt{\int_{\Omega} \iint_{\Sigma}\left|\mathbf{G}^{E J}\left(x \mid x^{\prime}\right) \cdot \mathbf{E}\left(x^{\prime}\right)\right|^{2} d s d \omega}
\end{array}
$$

Each green functions are calculated from FDTD simulation. In this study, we use three components of received electric fields and one components of transmitted electric current to calculate model gradient. Step length of model parameter is determined by subspace method $^{5)}$.

\section{RESULTS AND DISCUSSIONS}

In this study, we assume model as Fig.3.a and Fig.3.b. A transmitter and seven receivers are arranged above the anomaly. Electric current is transmitted while the transmitter moves to seven positions. Totally, the forward calculation is performed seven times to calculate one gradient in each iteration. We set the waveform as ricker wavelet with center frequency as $1 \mathrm{~Hz}$. We used 20 numbers of frequencies from $0 \mathrm{~Hz}$ to 4.9 Hz. We also set the grid size as $30 \mathrm{~m}$ and the number of grid is 31 in each directions. The initial model is set as two layer model.

Numerical results are shown in Fig.3.a, 3.b, 3.c, 3.d. In these results, we set the orientations of transmitter as x-oriented in Fig.3.a and as y-oriented in Fig.3.b, as z-oriented in Fig.3.c. In Fig.3.d, we consider three- 


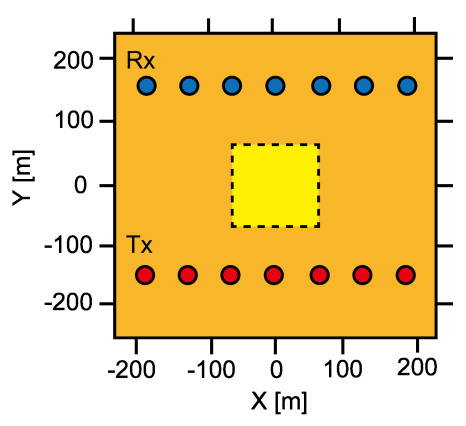

(a) Plane View

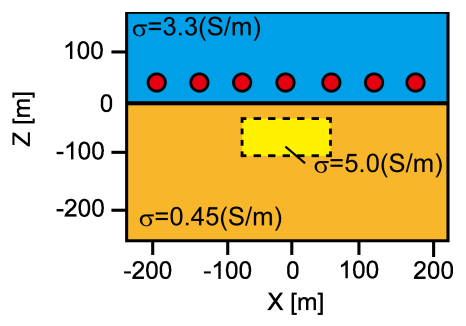

(b) Cross section

Fig. 4 Model configuration simulated in forward and inversion.

components of transmitter simultaneously. In each figure, a rectangle shows the existence area of conductive anomaly. Fig.7 shows vertical profile of inversion results in center of models $(X=450 \mathrm{~m}, \mathrm{Y}=450 \mathrm{~m})$. Fig. 6 shows horizontal profile of inversion results in Y:450m, $\mathrm{Z}: 360 \mathrm{~m}$.

From Fig.6, inversion results of Z-oriented transmitter have high resolution for vertical position of anomaly. From Fig.7, inversion results of $\mathrm{X}$ and Y- oriented transmitter have high resolution for horizontal position of anomaly. These differences of the inversion results are explained considering distribution of electric flux. In case of Fig.3.a and Fig.3.b, Fig.3.d, the horizontal flow of an electric flux around the anomaly is dominant. This is because the horizontal position of anomaly is resolved precisely. In case of Fig.3.c and Fig.3.d, the vertical flow of an electric flux around the anomaly is dominant. This is because the deeper position of anomaly is resolved precisely. In each profile, Fig.3.d has high resolution compared to the inversion results of a single orientation.

\section{CONCLUSION}

A full waveform CSEM inversion with the fictitious wave domain method was developed and implemented for a synthetic model. In this research, we compared the effect of the orientation of dipoles, and discussed the resolution of conductive anomaly. From the results, we find that the resolution depends on the orientations of transmitter and receivers. When the horizontal dipoles are employed, the obtained inversion result has a resolution to horizontal direction. When the vertical dipoles are employed, the obtained inversion result has much resolution to vertical direction. We integrated inversion results of different oriented dipoles. As a result, resolution of anomaly is improved when three components of transmitter are employed. Through all numerical calculations, we conclude that it is important to consider different orientation of dipoles.

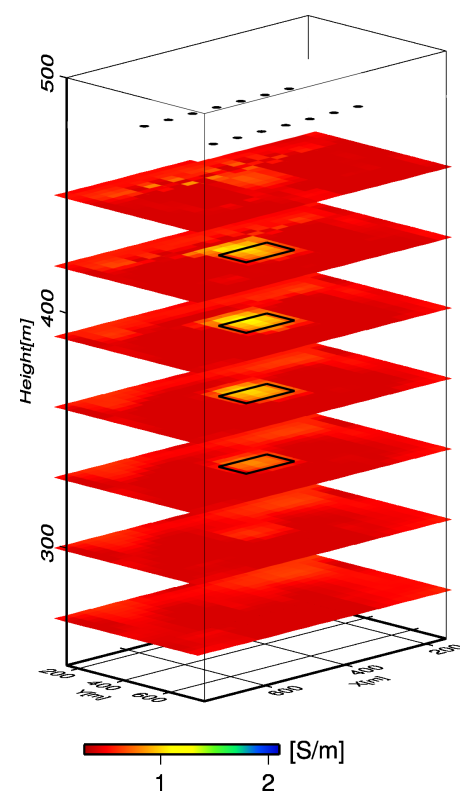

(a) X-oriented transmitter

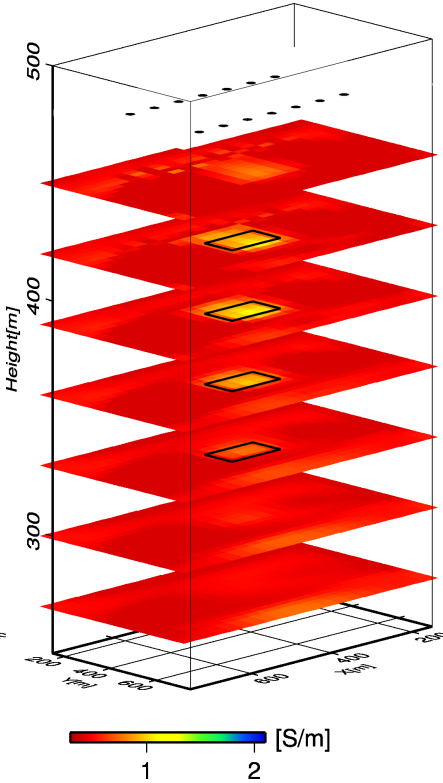

(b) Y-oriented transmitter

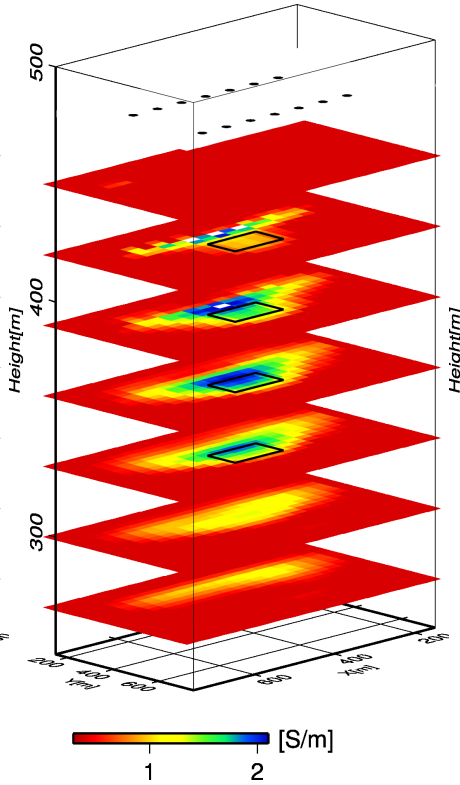

(c) Z-oriented transmitter

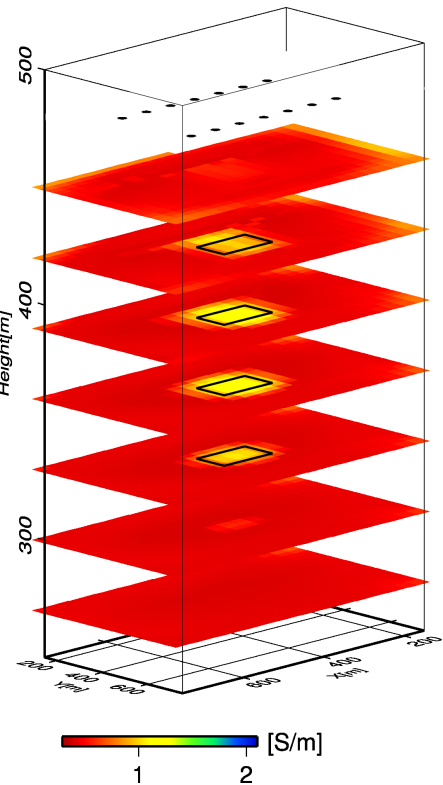

(d) X,Y,Z-oriented transmitter

Fig. 5 Comparison of inversion results changing the orientations of transmitter. 


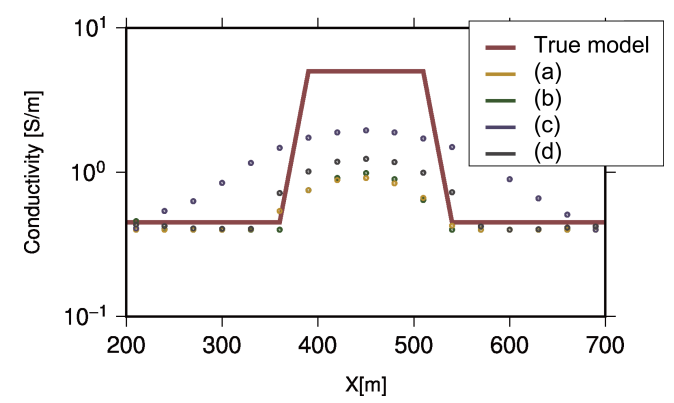

Fig. 6 Horizontal profile of inversion results in Y: $450 \mathrm{~m}, \mathrm{Z}: 360 \mathrm{~m}$.

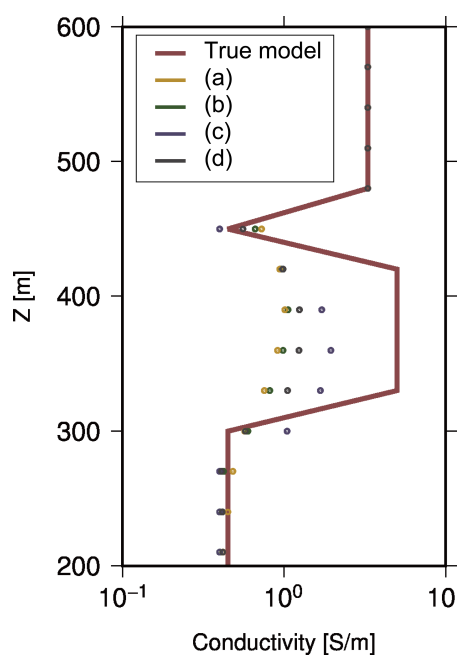

Fig. 7 Vertical profile of inversion results in $\mathrm{X}: 450 \mathrm{~m}, \mathrm{Y}: 450 \mathrm{~m}$.
ACKNOWLEDGEMENT We are greatly thankful to financial support of SMS exploration project by Ministry of Education, Culture, Sports, Science and Technology in Japan. We are also grateful to the Japan Society for the Promotion of Science (JSPS 24.372) for the Grants-in-Aid for Science Research in the Research Fellowship program that supported the present study.

\section{REFERENCES}

1) Zhdanov, M. S., and Portniaguine, O. (1997): Time - domain electromagnetic migration in the solution of inverse problems. Geophysical Journal International, 131, 2, 293-309.

2) Zhdanov, M. S., Velikhov, E. P., Cuma, M., Wilson, G., Black, N., Gribenko, A. (2010): Exploring multiple 3D inversion scenarios for enhanced interpretation of marine CSEM data: An iterative migration analysis of the Shtokman gas field: Barents Sea. First Break, 28, 3, 95-101.

3) Mittet, R. (2010): High-order finite-difference simulations of marine CSEM surveys using a correspondence principle for wave and diffusion fields. Geophysics, 75, 1, F33.

4) Ward, S. H., and G. W. Hohmann, 1987, Electromagnetic theory for geophysical applications: in M. N. Nabighian, ed., Electromagnetic methods in applied geophysics, Theory, 131-311.

5) Crase, E., Wideman, C, Noble, M. and Tarantola, A. (1992): Nonlinear elastic wave form inversion of land seismic reflection data, Journal of Geophysical Research, 97, 4658-4703. 